\title{
SwissDRG: mise au concours d'une étude concomitante
}

\author{
La FMH et $\mathrm{H}+$ lancent ensemble une mise au concours pour une étude scientifique \\ sur les transferts de prestations et de coûts entre le secteur stationnaire et le secteur \\ ambulatoire.
}

Beatrix Meyer

Cheffe du Service tarifaire de la FMH et du domaine SwissDRG
Correspondance: Beatrix Meyer Service tarifaire FMH Froburgstrasse 15 CH-4600 Olten Tél. 0313591111 swissdrg@fmh.ch
Depuis plusieurs années, la FMH ne cesse de souligner l'importance d'une recherche concomitante à l'occasion de l'introduction de SwissDRG. Les recommandations concrètes à cet égard n'ont pas manqué. En 2009 déjà, la FMH a présenté un concept à SwissDRG SA pour la réalisation d'une recherche concomitante [1]. L'une des recommandations du concept de la FMH était d'étudier les transferts de prestations et de coûts entre le secteur stationnaire et le secteur ambulatoire (hôpitaux et cabinets inclus). Mais malheureusement, SwissDRG SA a décidé de ne pas assumer la responsabilité principale de cette recherche, si bien que les recommandations du concept de la FMH n'ont pas été appliquées.

Après cette décision de SwissDRG SA, le corps médical espérait que l'Office fédéral de la santé publique (OFSP) se pencherait sur la question. L'étude de faisabilité de l'OFSP, visant à évaluer la révision de la LAMal, propose qu'un des 15 projets partiels étudie les transferts de coûts du secteur stationnaire au secteur ambulatoire [2]. Cependant, la décision de l'OFSP de passer à la réalisation de cette étude est attendue depuis l'été 2010. Et même si l'OFSP finit par se décider à analyser cet aspect important, la méthode prévue ne répondra pas aux besoins des prestataires de soins. Et pour cause, les auteurs de l'étude de faisabilité de l'OFSP recommandent de ne commencer le projet qu'en 2013. De plus, ils suggèrent une analyse secondaire bien que les données nécessaires à cet effet manquent dans le domaine ambulatoire hospitalier.

Qui va donc maintenant se pencher sur la question - essentielle pour le corps médical - des transferts de coûts et de prestations entre le secteur stationnaire de soins aigus et le secteur ambulatoire des hôpitaux et cabinets privés? Devons-nous attendre en vain, à l'instar de nos collègues allemands, et craindre qu'une telle étude arrive trop tard? Pour éviter ce scénario, la FMH a ouvert le dialogue avec $\mathrm{H}+$. De concert, ils ont décidé de mettre au concours une étude scientifique pour analyser la question. Les intéressés trouveront les informations à ce sujet sous www.fmh.ch $\rightarrow$ Tarifs $\rightarrow$ SwissDRG $\rightarrow$ Recherche concomitante. Ainsi, l'étude débutera certes tardivement, mais avant l'introduction de SwissDRG.
L'étude devra non seulement établir le volume de transfert éventuel d'investigations et de traitements $\mathrm{du}$ secteur stationnaire de soins aigus vers le secteur ambulatoire (hôpital et pratique privée), mais aussi déterminer quelles en sont les raisons. Quelle est ici l'influence du système DRG? Quelle est par ex. l'influence du développement de la chirurgie de jour? Grâce à cette étude, les prestataires de soins disposeront à l'avenir de données fiables et seront en mesure d'argumenter sur la base de chiffres et de faits face aux responsables politiques et aux partenaires. En outre, il faudra veiller à ne pas assimiler à tort les éventuelles réductions des durées d'hospitalisation et les transferts d'examens et de traitements vers le secteur ambulatoire à une «augmentation des prestations».

Toutefois, cette analyse de même que l'étude lancée par la FMH sur l'évolution de la relation médecinpatient et des conditions-cadres des médecins hospitaliers [3] ne mettent en œuvre qu'une infime partie de ce que représente la recherche concomitante demandée par la FMH [1]. Dans ce contexte, la FMH et $\mathrm{H}+$ doivent se concentrer sur les thèmes les plus importants pour leurs membres. Mais il reste nécessaire que tous les partenaires du domaine de la santé conjuguent leurs efforts pour atteindre l'objectif d'un monitorage à large échelle, garant d'une politique de la santé durable.

\section{Références}

1 Bovier P, Burnand B, Guillain H, Paccaud F, Vader JP, Locher H, Meyer B. Concept pour la recherche concomitante à l'occasion de l'introduction de SwissDRG, www.fmh.ch $\rightarrow$ Tarifs $\rightarrow$ SwissDRG $\rightarrow$ Recherche concomitante; 2009.

2 Pellegrini S, Widmer T, Weaver F, Fritschi T, Bennett J. Révision de la LAMal, financement des hôpitaux: étude de faisabilité et développement d'un concept pour une future évaluation. Rapport final, sur mandat de l'Office fédéral de la santé publique www. bag.admin.ch/evaluation/01759/07350/07641/index. html?lang=fr, 2010. p. 82s.

3 Etude concomitante à l'occasion de l'introduction de SwissDRG: Evolution de la relation médecin-patient et des conditions-cadres pour les médecins hospitaliers, cf. www.fmh.ch $\rightarrow$ Tarifs $\rightarrow$ SwissDRG $\rightarrow$ Recherche concomitante. 